\title{
IL6 trans-signaling associates with ischemic stroke but not with atrial fibrillation
}

\author{
Louise Ziegler ${ }^{1 *}$, Håkan Wallén², Sara Aspberg², Ulf de Faire ${ }^{3}$ and Bruna Gigante ${ }^{4}$
}

\begin{abstract}
Background: Pro-inflammatory processes underlie ischemic stroke, albeit it is largely unknown if they selectively associate with the risk of atherothrombotic or cardioembolic ischemic stroke. Here we analyze whether pro-inflammatory interleukin (IL) 6 trans-signaling, is associated with the risk of ischemic stroke and underlying atrial fibrillation (AF).

Methods: During a 20-year follow-up, 203 incident ischemic strokes were recorded from national registers in the cohort of 60-year-old men and women from Stockholm $(n=4232)$. The risk of ischemic stroke associated with circulating IL6 trans-signaling, assessed by a ratio between the pro-inflammatory binary IL6:SIL6R complex and the inactive ternary IL6:SIL6R:sgp130 complex (B/T ratio), was estimated by Cox regression and expressed as hazard ratio (HR) with a 95\% confidence interval (CI) in the presence or absence of AF. Risk estimates were adjusted for cardiovascular risk factors and anticoagulant treatment. In a secondary analysis, the association of IL6 trans-signaling with the risk of incident AF $(n=279)$ was analyzed.
\end{abstract}

Results: $\mathrm{B} / \mathrm{T}$ ratio $>$ median was associated with increased risk of ischemic stroke in study participants without $\mathrm{AF}$ (adjusted HR 1.49; 95\% Cl 1.08-2.06), while an association could not be demonstrated in the presence of AF. Moreover, the $\mathrm{B} / \mathrm{T}$ ratio was not associated with the risk of $\mathrm{AF}$ (HR 0.96; $95 \% \mathrm{Cl} 0.75-1.24)$.

Conclusions: Pro-inflammatory IL6 trans-signaling, estimated by the B/T ratio, is associated with ischemic stroke in individuals without AF. These findings suggest that the $B / T$ ratio could be used to assess the risk of non-AF associated ischemic stroke.

Keywords: Epidemiology, Ischemic stroke, Atrial fibrillation, Inflammation

\section{Background}

Inflammation driven by interleukin (IL) 6 represents one of the mechanisms underlying different forms of ischemic stroke [1-3]. IL6 signals through two pathways mediating opposing effects. In trans-signaling, IL6 mediates a potent pro-inflammatory and pro-atherogenic effect while classical IL6 signaling entails effects essential to the immune system and tissue homeostasis [4]. In classical signaling, IL6 binds the membrane bound IL6 receptor (IL6R) and the signal transducing receptor, glycoprotein

\footnotetext{
*Correspondence: louise.dencker-ziegler@sll.se

${ }^{1}$ Department of Clinical Sciences Karolinska Institutet, Division of Internal

Medicine, Danderyd Hospital, S-182 88 Stockholm, Sweden

Full list of author information is available at the end of the article
}

130 (gp130). In IL6 trans-signaling on the other hand, IL6 binds a soluble IL6R isoform (sIL6R) forming the circulating IL6:sIL6R (binary) complex able to bind and activate gp130. The active binary IL6:sIL6R complex is inhibited by the soluble gp130 (sgp130) through the rapid formation of an IL6:sIL6R:sgp130 (ternary) complex. The ternary complex impedes IL 6 trans-signaling by preventing binding to gp130 [5].

IL6 trans-signaling contributes to sustained inflammation in chronic conditions such as atherosclerosis $[4,6]$ and experimental research indicate that transsignaling could be detrimental in the ischemic brain [7]. We recently demonstrated that IL6 trans-signaling is associated with the risk of ischemic stroke and the binary/ternary complex $(\mathrm{B} / \mathrm{T})$ ratio, improved risk 
classification measures in individuals otherwise classified as at low-intermediate risk for cardiovascular events [8]. We have also shown that all IL6 trans-signaling components (IL6, sIL6R and sgp130) are expressed in high-grade carotid artery plaques indicating a role for IL6 trans-signaling in large vessel cerebrovascular disease [9].

Biomarkers of IL6 trans-signaling are emerging in cardiovascular risk prediction $[6,10-12]$. However, there are no studies addressing their role as predictors of cardioembolic vs. atherothrombotic ischemic stroke. As the preventive treatment modalities in ischemic cerebrovascular disease differ, novel biomarkers able to improve prediction of cardioembolic and atherothrombotic stroke would be of great clinical relevance. Atrial fibrillation (AF), the most common supraventricular arrhythmia is associated with an increased risk of cardiac embolization and ischemic stroke and the inflammatory state described in AF includes higher plasma levels of IL6 associated with an increased risk of secondary thromboembolism and mortality [13-15]. IL6 trans-signaling specifically has however not been studied in relation to AF or cardioembolic stroke risk.

\section{Aims}

We aimed at investigating the role of IL6 trans-signaling in ischemic stroke in relation to AF. The primary aim was to analyze a potential association between IL6 trans-signaling, mirrored by the $\mathrm{B} / \mathrm{T}$ ratio, and the risk of ischemic stroke in a prospective cohort of middle-aged subjects free of prevalent cardiovascular disease (CVD) with and without AF. The secondary aim was to analyze possible associations between IL6 trans-signaling, and the risk of incident AF.

\section{Methods}

\section{Study population}

In the cohort study of 60-year-old men and women from Stockholm, every third man and woman living in the Stockholm County and turning 60 years in 19971998 was invited to participate and, with a $78 \%$ positive response rate, 4232 participants were included [6]. At the baseline visit, participants were given a self-administered questionnaire on lifestyle, medical family history, past and chronic diagnoses and current medication. Body weight, height and blood pressure was measured, a 12-lead resting electrocardiogram (ECG) recorded and fasting blood samples drawn in the morning and immediately frozen to -80 degrees Celsius for future analyses. Subjects with signs of infection were rescheduled to a later date to avoid the inflammatory markers from being affected.
Subjects were excluded from the present analysis if they had not filled out the questionnaire $(n=122)$, lacked serum samples $(\mathrm{n}=96)$, had prevalent coronary or cerebrovascular disease at baseline $(n=369)$ or incident coronary events during follow-up $(n=433)$. In addition, participants inaccurately categorized as incident ischemic stroke with the International Classification of Diseases 10th revision (ICD-10) diagnosis codes I649 or I652 $(\mathrm{n}=19)$ were restricted from final analyses leaving 3193 individuals in the primary analysis. For a detailed overview of the exclusions please see Additional Fig. 1.

\section{Outcome ascertainment}

Study participants were followed up until December $31^{\text {st }}$, 2017 via their personal identification numbers through linkage to the Swedish national registers; The Swedish National Inpatient Register with a $100 \%$ capture of all hospitalized patients in Sweden and The National Cause of Death Register recording all deaths and cause of death diagnoses in Sweden. Primary diagnoses of non-fatal and fatal ischemic stroke (I63) were registered. In secondary analyses, main and secondary diagnoses of incident AF (I48) were registered to assess incident AF. The ICD-10 code I489 includes atrial flutter, i.e. cases of atrial flutter were included. To analyze the risk of incident AF, subjects with prevalent AF were excluded $(n=29)$ as were incident ischemic stroke cases $(n=198)$ due to the wellknown high proportion of undiagnosed AF in this group (Additional Fig. 1).

\section{Biochemical measurements and derivation of the binary and ternary complex molar concentrations}

Baseline serum levels of IL6, sIL6R and sgp130 were analyzed as described in the Additional files and in a prior publication [6]. The binary (IL6:sIL6R) and ternary (IL6:sIL6R:sgp130) complexes were estimated from their molar concentrations with formulas previously presented $[6,16,17]$. The ratio between the binary and ternary complex, $\mathrm{B} / \mathrm{T}$ ratio was calculated for each individual.

\section{Statistical analysis}

Continuous variables are presented as median and interquartile range (IQR) while binary variables are presented as numbers and percentages.

The risk of ischemic stroke associated with IL6 transsignaling estimated by the $\mathrm{B} / \mathrm{T}$ ratio was analyzed by Cox proportional hazards model. The risk estimates are given as hazard ratios (HR) with $95 \%$ confidence intervals $(\mathrm{CI})$. In the primary analysis, the risk of ischemic stroke was analyzed in subgroups defined by the presence or absence of AF (prevalent or incident). The results from the primary analysis are presented in a crude model and after adjustment for the common 
cardiovascular risk factors identified at baseline: sex, body mass index (BMI) expressed as $\mathrm{kg} / \mathrm{m}^{2}$, hypertension (self-reported, or blood pressure $>140 / 90 \mathrm{~mm} \mathrm{Hg}$ recorded at the baseline visit), diabetes mellitus (selfreported, or fasting glucose $>7.0 \mathrm{mmol} / \mathrm{L}$ in the baseline test), hypercholesterolemia (self-reported or fasting total cholesterol $>5.0 \mathrm{mmol} / \mathrm{L}$ ), smoking, and chronic treatment at baseline with anticoagulant drugs with codes from the Anatomic Therapeutic Chemical classification system (ATC): B01AA (vitamin K antagonists) or B01AB (heparin group).

In secondary analyses, the risk of incident AF associated with IL6 trans-signaling was analyzed, including each of the IL6 trans-signaling components IL6, sIL6R, sgp130, and the $\mathrm{B} / \mathrm{T}$ ratio categorized in quartiles or dichotomized at the median. The secondary analysis is presented in a crude model and in a model adjusted for sex, hypertension, BMI, and left ventricular hypertrophy defined as the presence of either one of two established ECG criteria, the Minnesota Code or Cornell voltageduration product.

To account for the effect of age on the risk of AF, the cumulative AF incidence was also presented graphically using Kaplan Meier curves and stratified by the $\mathrm{B} / \mathrm{T}$ ratio dichotomized at the median and in additional analyses stratified by quartiles of IL6, sIL6R and sgp130 and for IL6 also dichotomized at the $75^{\text {th }}$ percentile. Log-rank test was used to test for equality in the survivor functions. To estimate the difference in time to AF diagnosis, quantile regression for censored data was implemented using Laplace regression analysis, expressed in years with $95 \%$ $\mathrm{CI}$ and adjusted for the above-mentioned confounders.

All statistical analyses were performed on Stata statistical software, Release 14. College Station, TX: StataCorp LP.

\section{Results}

In Table 1, the clinical characteristics of the study populations are presented stratified by the occurrence of an ischemic stroke during follow-up or not. As expected, those that suffered an ischemic stroke carried a greater cardiovascular risk burden than those who did not. Moreover, stroke cases had a significantly higher $\mathrm{B} / \mathrm{T}$ ratio at baseline compared to non-cases $(p=0.0003)$.

During an approximately 20 -year follow-up, there were 203 fatal and non-fatal cases of ischemic stroke. Prevalent AF was registered in 29 study participants at baseline and incident AF in 279 participants during follow-up. Two of the participants with AF at baseline were on warfarin prophylaxis and five suffered an ischemic stroke during follow-up. Of the incident AF cases, 161 were diagnosed with $\mathrm{AF}$ as a main diagnosis, 116 as a secondary diagnosis and two had an AF diagnosis not categorized as main or secondary. Study participants with AF (prevalent or incident) suffered more ischemic strokes during followup than those without $\mathrm{AF}$ while levels of the $\mathrm{B} / \mathrm{T}$ ratio did not differ (Table 2).

Risk of future ischemic stroke associated with the $B / T$ ratio in subjects with and without atrial fibrillation

Figure 1 presents graphically the risk of future ischemic stroke associated with the $\mathrm{B} / \mathrm{T}$ ratio $>$ median (1.58) in subjects without AF (adjusted HR 1.49; 95\% CI 1.082.06 ) and in those with prevalent or incident AF (adjusted HR 1.54; 95\% CI 0.81-2.91). Crude and adjusted risk estimates are presented in Additional Table 1.

\section{Risk of incident atrial fibrillation and time to atrial fibrillation associated with the B/T ratio and IL6 trans-signaling components}

There was no increased risk of incident AF associated with the $\mathrm{B} / \mathrm{T}$ ratio categorized into quartiles or dichotomized at the median (Table 3). There was no difference in cumulative incidence of AF when stratifying by the $\mathrm{B} / \mathrm{T}$ ratio cut at the median, $\mathrm{p}=0.87$ (Fig. 2).

Investigating the association with IL6 signaling further, there was a borderline significant association between the risk of AF and IL6 $>75^{\text {th }}$ percentile (HR 1.23; CI 0.931.63), as seen in Additional Table 2. In addition, a pattern of earlier AF diagnosis associated with high IL6 levels was seen in Kaplan Meier curves (Additional Fig. 2). At the end of follow-up, when adjusting for the above-mentioned confounders (sex, BMI, smoking, hypertension, diabetes, hypercholesterolemia and chronic treatment at baseline with anticoagulants), participants with IL6 levels $>75^{\text {th }}$ percentile were diagnosed with AF 4.3 years earlier (95\% CI $1.1-7.5$ years, $\mathrm{p}=0.008$ ) compared to those with lower levels. No association with increased risk of $\mathrm{AF}$ or earlier AF diagnosis was observed for the soluble trans-signaling receptors, sIL6R or sgp130 (Additional Table 3, Additional Fig. 3).

\section{Discussion}

Here we demonstrate that IL6 trans-signalling, mirrored by the $\mathrm{B} / \mathrm{T}$ ratio $>$ median, is associated with increased risk of ischemic stroke albeit the association could not be shown in the small group of participants with AF nor with the risk of AF specifically.

Increased IL6 levels in the circulation and in the central nervous system have been demonstrated in the acute phase of ischemic stroke $[18,19]$. The role of IL6 is however controversial with evidence of both detrimental and protective properties in ischemic stroke models and no effect on infarct size or neurological function in IL6 knock-out mice [20, 21]. 


Subgroups
Fig. 1 Risk of future ischemic stroke associated with the B/T ratio > median in subjects with and without a diagnosis of AF analyzed by Cox
regression and expressed as hazard ratio with 95\% confidence interval. Adjustments were made for sex, smoking, hypertension, hyperlipidemia,
diabetes mellitus, BMl, and antithrombotic treatment

Table 1 Baseline characteristics of the study population stratified by ischemic stroke

\begin{tabular}{lll}
\hline $\begin{array}{l}\text { Baseline clinical } \\
\text { characteristics }\end{array}$ & $\begin{array}{l}\text { Ischemic stroke } \\
(\mathbf{n = 2 0 3 )}\end{array}$ & $\begin{array}{l}\text { No ischemic stroke } \\
(\mathbf{n}=\mathbf{2 9 9 0})\end{array}$ \\
\hline Male (\%) & $116(57.1)$ & $1309(43.8)$ \\
Hypertension (\%) & $45(22.2)$ & $440(14.7)$ \\
Hyperlipidemia (\%) & $6(3.0)$ & $103(3.4)$ \\
Diabetes mellitus (\%) & $10(4.9)$ & $76(2.5)$ \\
Atrial fibrillation (\%) & $5(2.5)$ & $23(0.8)$ \\
Smoking (\%) & $56(27.9)$ & $589(20.0)$ \\
Anticoagulant treatment (\%) & $1(0.5)$ & $11(0.4)$ \\
BMl & $26.5(24.4-28.6)$ & $26.0(23.7-28.8)$ \\
Systolic blood pressure & $143.5(130.5-156.5)$ & $134.5(120.5-150)$ \\
Diastolic blood pressure & $88(81-94.5)$ & $82.5(76-90)$ \\
Glucose & $5.2(4.8-5.7)$ & $5.2(4.8-5.6)$ \\
LDL & $4.1(3.4-4.7)$ & $3.8(3.2-4.5)$ \\
B/T ratio & $1.60(1.56-1.63)$ & $1.58(1.55-1.61)$
\end{tabular}

Continuous variables are presented as median (IQR). BMI is expressed as $\mathrm{kg} / \mathrm{m}^{2}$, blood pressure as $\mathrm{mm} \mathrm{Hg}$, glucose and LDL as mmol/L. Missing data: Smoking $(n=40), L D L(n=37)$, and blood pressure $(n=3)$

We previously demonstrated that the $\mathrm{B} / \mathrm{T}$ ratio, a biomarker assessing the pro-inflammatory IL6 trans-signaling pathway, could predict an increased risk of incident ischemic stroke, pre-eminently in individuals with low LDL cholesterol [8]. Moreover, in a small "real-world" cohort of patients with carotid artery stenosis undergoing carotid endarterectomy $(n=78)$, we analyzed the expression of IL6 and IL6 receptor genes in carotid artery
Table 2 Number of ischemic strokes and $B / T$ ratio level in subjects with and without atrial fibrillation

\begin{tabular}{llll}
\hline & Never AF & AF & P \\
\hline Total number & 2748 & 445 & - \\
Stroke (\%) & $140(5 \%)$ & $63(14 \%)$ & $<0.0001$ \\
B/T ratio & $1.58(1.54-1.61)$ & $1.58(1.54-1.61)$ & 0.94
\end{tabular}

Number of participants with ischemic stroke during follow-up (\%) and levels of the $B / T$ ratio presented as median (IQR) in subjects with $A F$ (prevalent or incident) compared to those without (Never AF)

Table 3 Risk of incident atrial fibrillation associated with the B/T ratio

\begin{tabular}{llclc}
\hline $\mathbf{B} / \mathbf{T}$ ratio & Crude & $\mathbf{P}$ & Adjusted & $\mathbf{P}$ \\
\hline$\leq 25^{\text {th }}$ perc & $1.00($ ref $)$ & - & $1.00($ ref $)$ & \\
$25-50^{\text {th }}$ perc & $0.93(0.71-1.23)$ & 0.65 & $0.89(0.67-1.17)$ & 0.41 \\
$50-75^{\text {th }}$ perc & $1.01(0.77-1.32)$ & 0.93 & $0.92(0.70-1.21)$ & 0.57 \\
$>75^{\text {th }}$ perc & $1.00(0.77-1.32)$ & 0.94 & $0.86(0.65-1.14)$ & 0.30 \\
$\leq$ median & $1.00($ ref $)$ & - & $1.00($ ref $)$ & - \\
$>$ median & $1.04(0.86-1.26)$ & 0.67 & $0.94(0.78-1.15)$ & 0.59 \\
\hline
\end{tabular}

Risk of incident $A F$ associated with the $B / T$ ratio, categorized into percentiles (perc) and dichotomized at the median, analyzed by Cox regression and expressed as $\mathrm{HR}(95 \% \mathrm{Cl})$. Multivariate analysis adjusted for sex, hypertension, $\mathrm{BMI}$, and left ventricular hypertrophy. Participants with prevalent AF at baseline were excluded from this analysis. Missing data on left ventricular hypertrophy $(n=6)$ 


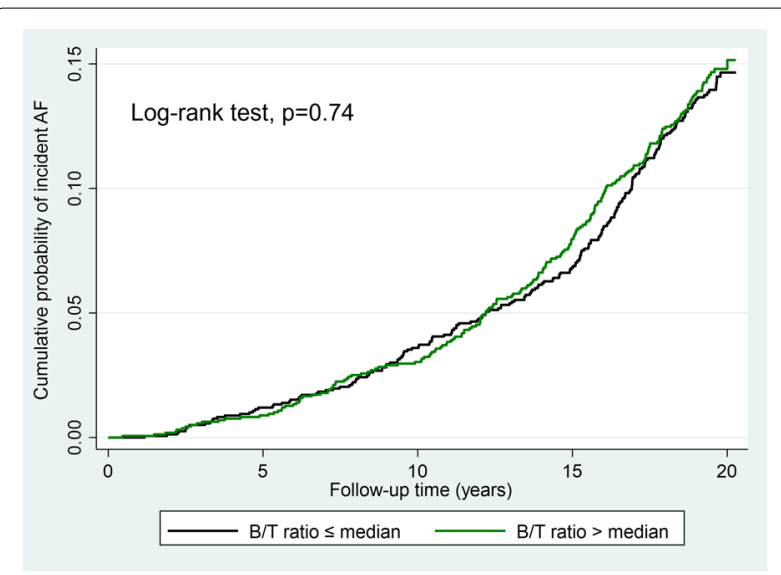

Fig. 2 Cumulative incidence of AF in subjects without prevalent AF at baseline stratified by the $B / T$ ratio dichotomized at the median

plaques and found that their expression were upregulated in patients with a recent ischemic cerebrovascular event ( $\leq 6$ months from carotid surgery) [9]. Our former and current data suggest that the IL6 trans-signaling pathway is mainly associated with atherosclerosis related cerebrovascular disease as we could not demonstrate an association with the risk of AF or an increased risk of ischemic stroke in subjects with AF. Of note is that the prevalence and risk factors for AF overlap those for large and small vessel cerebrovascular disease i.e. the underlying pathophysiology is not by default cardioembolic in individuals with AF [22]. A possible implication of our findings is however that the $\mathrm{B} / \mathrm{T}$ ratio may represent a biomarker to identify individuals at risk for atherothrombotic but not cardioembolic stroke.

Results from Mendelian randomization studies indicate a causal association between the $I L 6 R, \mathrm{AF}$ and ischemic stroke and that the increased risk of ischemic stroke associated with the IL6R is accounted for by AF [23, 24]. A more recent Mendelian randomization study on the other hand found an association between IL6R and the risk of large vessel stroke but not cardioembolic stroke [25]. Nevertheless, these studies do not consider the differential role of IL6 classical and IL6 trans-signaling in the modulation of inflammatory processes, and that the synthesis and release of sIL6R and sgp130 is regulated at a post-transcriptional level. Whether IL6 classical or IL6 trans-signaling is associated with AF or not has not been analyzed in epidemiological studies to this date. Our data suggest that IL6 is possibly associated with an increased risk of AF and that this association is not mediated by IL6 trans-signaling, but potentially driven by the IL6 classical signaling. The observation that there was no difference in the $\mathrm{B} / \mathrm{T}$ ratio with regards to $\mathrm{AF}$, as well as the fact that neither sIL6R nor sgp130 serum levels were associated with the risk of AF; suggest that the stroke risk associated with the $\mathrm{B} / \mathrm{T}$ ratio is independent of $\mathrm{AF}$.

\section{Strengths and limitations}

This is the first prospective population-based cohort study exploring the association between the pro-inflammatory IL6 trans-signaling and ischemic stroke in relation to AF. The unique Swedish personal identification number and mandatory reporting of inpatient and hospital-based outpatient diagnoses in national Swedish registers enable a $100 \%$ follow-up. With an overall positive predictive value of diagnoses in these registers of 85-95\%, diagnoses may be considered reliable.

One major limitation in the study is the lack of diagnoses from primary care in the Swedish inpatient registers. In relation to ischemic stroke this is a marginal problem as stroke is mainly diagnosed in hospitals. AF however is also diagnosed in primary care. To account for this, we have recorded both main and secondary diagnoses of AF, but we cannot entirely compensate for the lack of primary care diagnoses. In addition, as AF in the early stages of the disease is paroxysmal and often asymptomatic, potential delays in diagnosing the condition is impending and thus the first recorded diagnosis is not always equal to the first presentation of the disease. Furthermore, we did not perform long-term ECG recordings in the study, possibly missing paroxysmal AF which it problematic as even subclinical atrial tachyarrhythmias are associated with an increased risk of ischemic stroke [26]. In addition, we did not have enough power to analyze differences in association between pre- and post-stroke AF. Moreover, in the present study we aimed at analyzing the risk of ischemic stroke associated with IL6 trans-signaling in individuals with and without AF. Without access to information on findings from computer tomography/ magnetic resonance imaging, carotid ultrasound, longterm ECG recordings, echocardiogram, etc. we were forced to use the composite outcome of ischemic stroke despite its heterogeneous pathophysiological mechanisms and can thus merely speculate on whether the association seen is primarily driven by atherothrombotic rather than cardioembolic origin.

Misclassification would however lead to an underestimation of AF prevalence and incidence and cardioembolic strokes in our cohort and would marginally affect our results: misclassification of ischemic stroke as atherothrombotic could possibly dilute the observed risk since the $\mathrm{B} / \mathrm{T}$ ratio was associated neither with $\mathrm{AF}$ nor with ischemic stroke in AF patients. The non-significant association with stroke risk in the smaller group of 
participants with prevalent/incident AF could however be due to lack of power albeit the risk estimate was comparable to that in the non-AF group.

In addition, we did not have access to information on prospective changes in medication. With the primary aim to analyze the $\mathrm{B} / \mathrm{T}$ ratio as a predictive marker of ischemic stroke this is however not mandatory.

Moreover, we only have serum samples from baseline and thus cannot exclude that serum levels at baseline do not mirror the levels during follow-up. In addition, the cut-offs for the B/T ratio and IL6 are data driven which can prevent the results from being generalizable. The aim of the study is however not to find suitable reference values but to analyze associations between IL6 trans-signaling and ischemic stroke. On the other hand, we believe that the data derived from this cohort, being a population-based cohort with participants randomly chosen and with a high positive response rate $(78 \%)$, are representable in the context of other populations with comparable lifestyle and societal structure.

Finally, the study is observational preventing any mechanistic conclusions from being drawn. The experimental evidence underlying the hypothesis of this study is however in line with the present findings.

\section{Conclusions}

IL6 trans-signaling, assessed by the $\mathrm{B} / \mathrm{T}$ ratio, is associated with an increased risk of ischemic stroke in patients without known AF. The results suggest the $\mathrm{B} / \mathrm{T}$ ratio could be a novel biomarker for a more personalized ischemic stroke risk assessment.

\begin{abstract}
Abbreviations
AF: Atrial fibrillation; ATC: Anatomic Therapeutic Chemical classification system; BMI: Body mass index; $\mathrm{B} / \mathrm{T}$ ratio : Binary/ternary complex ratio; Cl: Confidence interval; CVD: Cardiovascular disease; ECG: Electrocardiogram; gp130: Glycoprotein 130; HR: Hazard ratio; ICD-10: International Classification of Diseases 10th revision; IL: Interleukin; IL6R: Interleukin 6 receptor; IQR: Interquartile range; sgp 130: Soluble glycoprotein 130; sIL6R: Soluble interleukin 6 receptor.
\end{abstract}

\section{Supplementary Information}

The online version contains supplementary material available at https://doi. org/10.1186/s12883-021-02321-6.

\section{Additional file 1.}

\section{Acknowledgements}

Not applicable.

\section{Authors' contributions}

$L Z, B G, H W$, and $U d F$ planned the design of the study. $L Z$ and $B G$ contributed to the data acquisition, performed the data analysis and drafted the manuscript. All authors contributed to the interpretation of the results, the critical revision of the manuscript and gave final approval of the work.

\section{Funding}

Open access funding provided by Karolinska Institute. This work was supported by the Stockholm County Council ALF project, Strategic research in Epidemiology at Karolinska Institutet and the Memorial Foundation of Sigurd and Elsa Golje (to BG). The funding bodies did not have any impact on the study design, nor on the analysis and interpretation of data or in drafting the manuscript.

\section{Availability of data and materials}

The datasets used and/or analyzed during the current study are available from the corresponding author on reasonable request.

\section{Declarations}

\section{Ethics approval and consent to participate}

Participants gave their informed consent before entering the study as previously described [6]. The study was approved by the local Ethical board at Karolinska Institutet (reference number 96-398) and abides to the Helsinki Declaration ethical principles for medical research involving human participants.

\section{Consent for publication}

Not applicable.

\section{Competing interests}

The authors declare that they have no competing interests.

\section{Author details}

${ }^{1}$ Department of Clinical Sciences Karolinska Institutet, Division of Internal Medicine, Danderyd Hospital, S-182 88 Stockholm, Sweden. ${ }^{2}$ Department of Clinical Sciences Karolinska Institutet, Division of Cardiovascular Medicine, Danderyd Hospital, Stockholm, Sweden. ${ }^{3}$ Unit of Cardiovascular and Nutritional Epidemiology Karolinska Institutet, Stockholm, Sweden. ${ }^{4}$ Cardiovascular Medicine Unit, Department of Medicine Karolinska Institutet, Stockholm, Sweden.

Received: 11 April 2021 Accepted: 7 July 2021

Published online: 09 August 2021

\section{References}

1. Okazaki S, Sakaguchi M, Miwa K, Furukado S, Yamagami H, Yagita Y, et al. Association of interleukin-6 with the progression of carotid atherosclerosis: a 9-year follow-up study. Stroke. 2014;45(10):2924-9. https://doi.org/ 10.1161/strokeaha.114.005991.

2. Fu Y, Yan Y. Emerging Role of immunity in cerebral small vessel disease. Front Immunol. 2018;9:67. https://doi.org/10.3389/fimmu.2018.00067.

3. Wu N, Xu B, Xiang Y, Wu L, Zhang Y, Ma X, et al. Association of inflammatory factors with occurrence and recurrence of atrial fibrillation: a metaanalysis. Int J Cardiol. 2013;169(1):62-72. https://doi.org/10.1016/j.ijcard. 2013.08.078.

4. Schuett $\mathrm{H}$, Oestreich R, Waetzig GH, Annema W, Luchtefeld M, Hillmer A, et al. Transsignaling of interleukin- 6 crucially contributes to atherosclerosis in mice. Arterioscler Thromb Vasc Biol. 2012;32(2):281-90. https://doi. org/10.1161/ATVBAHA. 111.229435.

5. Rose-John S. IL-6 trans-signaling via the soluble IL-6 receptor: importance for the pro-inflammatory activities of IL-6. Int J Biol Sci. 2012;8(9):1237-47. https://doi.org/10.7150/ijbs.4989.

6. Ziegler L, Gajulapuri A, Frumento P, Bonomi A, Wallen H, de Faire U, et al. Interleukin 6 trans-signalling and risk of future cardiovascular events. Cardiovasc Res. 2019;115(1):213-21. https://doi.org/10.1093/cvr/cry191.

7. Campbell IL, Erta M, Lim SL, Frausto R, May U, Rose-John S, et al. Transsignaling is a dominant mechanism for the pathogenic actions of interleukin-6 in the brain. The J Neurosc. 2014;34(7):2503-13. https://doi. org/10.1523/jneurosci.2830-13.2014.

8. Ziegler L, Frumento P, Wallen H, de Faire U, Gigante B. The predictive role of interleukin 6 trans-signalling in middle-aged men and women at low-intermediate risk of cardiovascular events. Euro J Prev Cardiol. 2020;27(2):122-9. https://doi.org/10.1177/2047487319869694. 
9. Ziegler L, Lundqvist J, Dreij K, Wallén $\mathrm{H}$, de Faire U, Paulsson-Berne G, et al. Expression of Interleukin 6 signaling receptors in carotid atherosclerosis. Vasc Med. 2021;26(1):3-10. https://doi.org/10.1177/1358863×20977662.

10. Santos RD, Rocha VZ. Bringing interleukin-6 complexity to preventive cardiology practice? Eur J Prev Cardiol. 2020;27(2):119-21. https://doi.org/ 10.1177/2047487319876765.

11. Ritschel VN, Seljeflot I, Arnesen H, Halvorsen S, Eritsland J, Fagerland MW, et al. Circulating levels of IL-6 receptor and gp130 and long-term clinical outcomes in ST-elevation myocardial infarction. J Am Heart Assoc. 2016;5:6. https://doi.org/10.1161/jaha.115.003014.

12. Groot HE, Hartman MH, Gu YL, de Smet BJ, van den Heuvel AF, Lipsic E, et al. Soluble interleukin 6 receptor levels are associated with reduced myocardial reperfusion after percutaneous coronary intervention for acute myocardial infarction. Cytokine. 2015;73(2):207-12. https://doi.org/ 10.1016/j.cyto.2015.02.004.

13. Guo Y, Lip GY, Apostolakis S. Inflammation in atrial fibrillation. J Am Coll Cardiol. 2012;60(22):2263-70. https://doi.org/10.1016/j.jacc.2012.04.063.

14. Conway DS, Buggins P, Hughes E, Lip GY. Relationship of interleukin-6 and C-reactive protein to the prothrombotic state in chronic atrial fibrillation. J Am Coll Cardiol. 2004;43(11):2075-82. https://doi.org/10.1016/j.jacc. 2003.11.062.

15. Aulin J, Siegbahn A, Hijazi Z, Ezekowitz MD, Andersson U, Connolly SJ, et al. Interleukin-6 and C-reactive protein and risk for death and cardiovascular events in patients with atrial fibrillation. Am Heart J. 2015;170(6):1151-60. https://doi.org/10.1016/j.ahj.2015.09.018.

16. Garbers C, Thaiss W, Jones GW, Waetzig GH, Lorenzen I, Guilhot F, et al. Inhibition of classic signaling is a novel function of soluble glycoprotein 130 (sgp130), which is controlled by the ratio of interleukin 6 and soluble interleukin 6 receptor. J Biol Chem. 2011;286(50):42959-70. https://doi. org/10.1074/jbc.M111.295758.

17. Muller-Newen G, Kuster A, Hemmann U, Keul R, Horsten U, Martens A, et al. Soluble IL-6 receptor potentiates the antagonistic activity of soluble gp130 on IL-6 responses. J Immunol. 1998;161(11):6347-55.

18. Tarkowski E, Rosengren L, Blomstrand C, Wikkelso C, Jensen C, Ekholm $S$, et al. Early intrathecal production of interleukin-6 predicts the size of brain lesion in stroke. Stroke. 1995;26(8):1393-8.
19. Loddick SA, Turnbull AV, Rothwell NJ. Cerebral interleukin-6 is neuroprotective during permanent focal cerebral ischemia in the rat. J Cerebral Blood Flow Metab. 1998;18(2):176-9. https://doi.org/10.1097/00004647199802000-00008.

20. Clark WM, Rinker LG, Lessov NS, Hazel K, Hill JK, Stenzel-Poore M, et al. Lack of interleukin-6 expression is not protective against focal central nervous system ischemia. Stroke. 2000;31(7):1715-20.

21. Suzuki S, Tanaka K, Suzuki N. Ambivalent aspects of interleukin-6 in cerebral ischemia: inflammatory versus neurotrophic aspects. J Cerebral Blood Flow Metab. 2009;29(3):464-79. https://doi.org/10.1038/jcbfm.2008.141.

22. Katsi V, Georgiopoulos G, Skafida A, Oikonomou D, Klettas D, Vemmos K, et al. Noncardioembolic Stroke in Patients with Atrial Fibrillation. Angiology. 2019;70(4):299-304. https://doi.org/10.1177/0003319718791711.

23. Schnabel RB, Kerr KF, Lubitz SA, Alkylbekova EL, Marcus GM, Sinner MF, et al. Large-scale candidate gene analysis in whites and African Americans identifies IL6R polymorphism in relation to atrial fibrillation: the National Heart, Lung, and Blood Institute's Candidate Gene Association Resource (CARe) project. Circulation Cardiovasc Genetics. 2011;4(5):55764. https://doi.org/10.1161/circgenetics.110.959197.

24. Rosa M, Chignon A, Li Z, Boulanger MC, Arsenault BJ, Bosse Y, et al. A Mendelian randomization study of IL6 signaling in cardiovascular diseases, immune-related disorders and longevity. NPJ Genomic Med. 2019;4:23; https://doi.org/10.1038/s41525-019-0097-4.

25. Georgakis MK, Malik R, Gill D, Franceschini N, Sudlow CLM, Dichgans M. Interleukin-6 signaling effects on ischemic stroke and other cardiovascular outcomes: a mendelian randomization study. Circ Genomic Precision Med. 2020;13:168-71. https://doi.org/10.1161/circgen.119.002872.

26. Healey JS, Connolly SJ, Gold MR, Israel CW, Van Gelder IC, Capucci A, et al. Subclinical atrial fibrillation and the risk of stroke. N Engl J Med. 2012;366(2):120-9. https://doi.org/10.1056/NEJMoa1105575.

\section{Publisher's Note}

Springer Nature remains neutral with regard to jurisdictional claims in published maps and institutional affiliations.
Ready to submit your research? Choose BMC and benefit from:

- fast, convenient online submission

- thorough peer review by experienced researchers in your field

- rapid publication on acceptance

- support for research data, including large and complex data types

- gold Open Access which fosters wider collaboration and increased citations

- maximum visibility for your research: over 100M website views per year

At BMC, research is always in progress.

Learn more biomedcentral.com/submissions 